\title{
Behavioral Change and Its Neural Correlates in Visual Agnosia After Expertise Training
}

\author{
Marlene Behrmann ${ }^{1}$, Jonathan Marotta ${ }^{2}$, Isabel Gauthier ${ }^{3}$, \\ Michael J. Tarr ${ }^{4}$, and Thomas J. McKeeff ${ }^{5}$
}

\begin{abstract}
Agnosia, the impairment in object and face recognition despite intact vision and intelligence, is one of the most intriguing, and debilitating, neuropsychological deficits. The goal of this study was to determine whether S.M., an individual with longstanding visual agnosia and concomitant prosopagnosia, can be retrained to perform visual object recognition and, if so, what neural substrates mediate this reacquisition. Additionally, of interest is the extent to which training on one type of visual stimuli generalizes to other visual stimuli, as this informs our understanding of the organization of ventral visual cortex. Greebles were chosen as the stimuli for retraining given that, in neurologically normal individuals, these stimuli can engage the fusiform face area. Posttraining,
\end{abstract}

\section{INTRODUCTION}

Visual agnosia refers to the well-known neuropsychological deficit in which recognition of visual stimuli is impaired despite intact vision and preserved semantic knowledge and intelligence (Farah, 2004). Although the severity of agnosia varies, so does the nature of the impairment; some individuals are impaired at recognizing objects as well as faces whereas others may be impaired at, for example, object and word recognition with relatively preserved face recognition. Exactly why these patterns of co-occurrence emerge is a matter of ongoing debate (Riddoch \& Humphreys, 2003; Buxbaum, Glosser, \& Coslett, 1999; Humphreys \& Rumiati, 1998; Rumiati \& Humphreys, 1997; Rumiati, Humphreys, Riddoch, \& Bateman, 1994; Farah, 1991, 1992, 1999). In addition to this debate, many other outstanding issues remain in the study of agnosia. One such issue, which is the focus of this article and which has received surprisingly little scientific attention, is whether agnosic individuals can be retrained to perform visual recognition. If such retraining is possible, 2 other immediate questions come to mind: The first concerns the extent and nature of the generalization from the training and

${ }^{1}$ Carnegie Mellon University, ${ }^{2}$ University of Manitoba, ${ }^{3}$ Vanderbilt University, ${ }^{4}$ Brown University, ${ }^{5}$ Princeton University
S.M. showed significant improvement in recognizing Greebles, although he did not attain normal levels of performance. He was also able to recognize untrained Greebles and showed improvement in recognizing common objects. Surprisingly, his performance on face recognition, albeit poor initially, was even more impaired following training. A comparison of preand postintervention functional neuroimaging data mirrored the behavioral findings: Face-selective voxels in the fusiform gyrus prior to training were no longer so and were in fact more Greeble-selective. The findings indicate potential for experience-dependent dynamic reorganization in agnosia with the possibility that residual neural tissue, with limited capacity, will compete for representations.

the second involves the cortical substrate that might mediate this reacquisition.

\section{Experience-dependent Change in Neuropsychological Deficits}

Whether experience-dependent change in visual agnosia is at all possible is of considerable interest in light of recent studies showing substantial compensation and recovery along with dynamic reorganization in damaged adult cortex (Kolb, Gibb, \& Robinson, 2003; Pizzamiglio, Galati, \& Committeri, 2001). For example, several studies document the recruitment of alternative neural tissue during the course of recovery from aphasia (Riecker, Wildgruber, Grodd, \& Ackermann, 2002) via activation of perilesional tissue in the lesioned hemisphere (Warburton, Price, Swinburn, \& Wise, 1999; Small, Flores, \& Knoll, 1998), via recruitment of homologous tissue in the opposite hemisphere (Thulborn, Carpenter, \& Just, 1999; Small et al., 1998), or both (Rosen et al., 2000). Similarly, many studies have documented plasticity of motor circuits in adults with hemiparetic stroke, providing support for a compensatory role of preexisting uncrossed motor neural pathways (Pizzamiglio et al., 2001).

Functional reorganization has also been noted in visual cortex in adults. For example, increased activation 
is observed in the visual cortex in blind individuals (Elbert \& Rockstroh, 2004), and learning-dependent changes in the corresponding retinotopic area of visual cortex are noted in normal observers following intensive monocular training on visual texture discrimination (Schwartz, Maquet, \& Frith, 2002). Of particular relevance are studies documenting behavioral and neural changes in individuals with lesions in visual cortex; there are several studies documenting a strong positive relationship between the recovery of visual function and improved metabolism of striate cortex (Pleger et al., 2003; Braus, Hirsch, Hennerici, Henn, \& Gass, 1999; Bosley et al., 1987), attesting to the efficacy of intervention procedures.

These findings suggest that dynamic cortical reorganization is possible in the human adult cortex. Few studies, however, are specifically devoted to tracking the effects of rehabilitation on agnosia and the associated cortical plasticity (Burns, 2004; Seniow, Polanowska, Mandat, \& Laudanski, 2003). Of the studies that have tracked recovery of individuals with agnosia, minimal, if any, change in the behavioral performance is reported. For example, Caldara et al. (submitted) reported the failure of a patient with a face recognition impairment (and preserved object recognition) to acquire expertise with visual input. Furthermore, in those few cases where change has been reported, it remains unclear whether the change is attributable to the adoption of compensatory strategies or to the amelioration of the object recognition deficit per se (Thomas, Forde, Humphreys, \& Graham, 2002). A primary goal of this study, then, is to examine the psychological and neural bases of experience-dependent change following focused intervention in an adult with longstanding visual agnosia and accompanying prosopagnosia.

\section{Implications of Retraining for Organization of the Ventral Visual Cortex}

Although the outcome of a study on recovery from visual agnosia is informative from a clinical point of view, such an intervention study can also increase our understanding of the organization of the cortical visual system. One issue that is currently debated in cognitive neuroscience concerns the organization of the ventral temporal visual system. Some researchers have suggested that this cortical region is organized along category-specific lines such that regions of cortex are selectively, perhaps even exclusively, dedicated to representing a specific type of visual object (such as faces, houses, or chairs). A particularly clear example of this comes from the domain of prosopagnosia, which, according to some views, is a consequence of damage to a face-specific region of ventral visual cortex (Farah, Rabinowitz, Quinn, \& Liu, 2000; Farah, 1990). This perspective is also compatible with the evidence from electrophysiological and functional imaging studies, doc- umenting a face-specific response in particular regions of the ventral cortex of the brain (Tsao, Freiwald, Knutsen, Mandeville, \& Tootell, 2003; Bentin \& Deouell, 2000; Kanwisher, 2000; Kanwisher, McDermott, \& Chun, 1997; McCarthy, Puce, Gore, \& Allison, 1997; Sergent, Ohta, \& MacDonald, 1992). An alternative conceptualization is that the ventral visual system is organized in a more distributed fashion with many different regions participating (perhaps to a greater or lesser extent) in the recognition of all visual stimuli (Pietrini et al., 2004; Haxby et al., 2001; Ishai, Ungerleider, Martin, Schouten, \& Haxby, 1999).

The controversy between a domain-specific organization of visual classes versus a more generic system has not been resolved and the reader is referred to recent articles for further explication (Grill-Spector, Knouf, \& Kanwisher, 2004; Grill-Spector, 2003; Tarr \& Cheng, 2003; Maurer, Le Grand, \& Mondloch, 2002; Haxby et al., 2001; Kanwisher, 2000; Tarr \& Gauthier, 2000). In this article, we implement category-specific retraining in an individual with object agnosia and prosopagnosia using one class of stimuli and then examine the extent to which there is generalization to other classes of stimuli. If different classes of objects are represented independently, perhaps by discrete modules, then one might not expect a trained class of stimuli to have any influence on a second, untrained class. In contrast, if retraining in one class has consequences for performance on a second class, this might implicate more general visual recognition mechanisms.

The training regimen we adopt involves the use of Greebles and has been previously shown to increase visual expertise in nonneurological individuals. In these studies (Gauthier, Williams, Tarr, \& Tanaka, 1998; Gauthier \& Tarr, 1997, 2002), participants are trained to identify individual Greebles, novel 3-D-rendered objects made of a vertically oriented "body" with 4 protruding appendages and sharing the same basic elemental features in a canonical configuration with other members of the class (see Figure 1). The claim is that, as is true for faces, local shape and surface features may not suffice for the purpose of discrimination and identification of individual Greebles given their perceptual similarity. To efficiently differentiate individual exemplars of faces or Greebles, additional details and "configural" or relational information may be necessary (Gauthier \& Tarr, 2002; Maurer et al., 2002). After expertise Greeble training, participants showed some of the hallmark patterns of recognition typically associated with faces such as inversion effects and sensitivity to configuration: Greeble parts were recognized more slowly in a transformed configuration than in the original configuration, but only when in the upright orientation (Gauthier \& Tarr, 1997, 2002). Interestingly, a neural correlate of this learning has been identified: After Greeble training, blood oxygen level-dependent (BOLD) activation was observed in the putative fusiform face area both for 


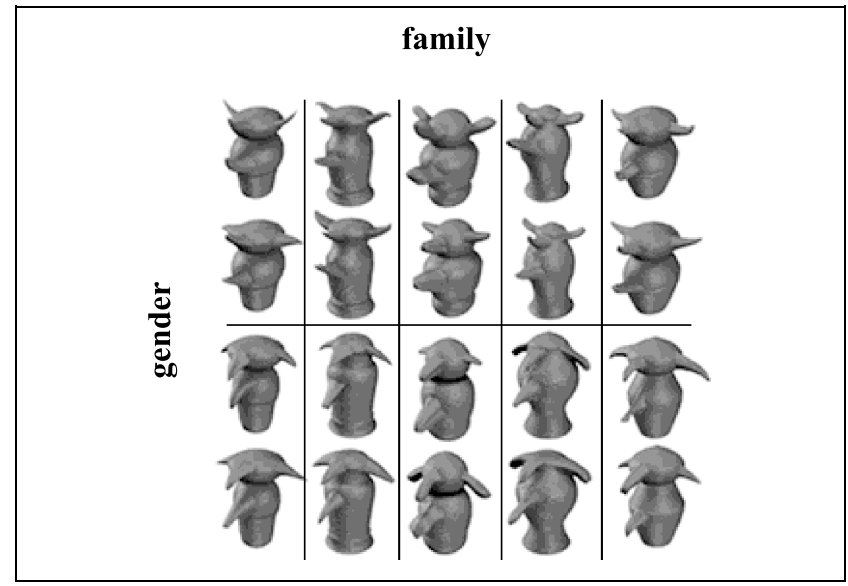

Figure 1. Examples of Greeble stimuli. Greebles are divided into families (columns), genders (major row division). Shown are examples of 2 individuals per family/sex combination. These Greebles are used in the generalization testing and in the imaging sessions, and similar stimuli are used for training.

faces and for Greebles (Gauthier \& Tarr, 2002; Gauthier, Tarr, Anderson, Skudlarski, \& Gore, 1999). These data support the view that a general-purpose recognition system in the ventral visual cortex may represent more than one stimulus class for which there is a demand for precise, configural knowledge.

Note that the need to differentiate individual exemplars within a class, and the subsequent development of configural representations, is evident in a host of other domains: Dog-show judges are impaired at recognizing inverted dogs (Diamond \& Carey, 1986) as are car and bird experts in their own domain of expertise (Gauthier, Skudlarski, Gore, \& Anderson, 2000). Consistent with the view of the fusiform area being recruited to mediate these learned visual representations, car and bird experts exhibited increased BOLD activation in the fusiform face area but only in response to stimuli in their domain of expertise (Gauthier et al., 2000). Moreover, greater activity was observed for cars than faces in the fusiform area of car experts who performed best on a behavioral measure of car expertise (Gauthier,
Curby, Skudlarski, \& Epstein, submitted; but see Rhodes et al., 2004).

Given the success of the Greeble training regimen in neurologically normal individuals, this study was designed to train an agnosic patient to recognize individual Greebles. The first question is whether the patient can benefit from the intervention at all, and if so, what neural correlates mediate the behavioral change. The second question is whether Greeble training would impact the patient's ability to recognize other visual stimuli, such as faces, as might be expected if finegrained recognition of both Greebles and faces rely on configural processing.

S.M., a 24-year-old man, with object agnosia and prosopagnosia of approximately 6 years' duration, is significantly debilitated by his face recognition deficit; his anecdotes include entering a supermarket with a family member, becoming separated, and then failing to recognize the person when reunited. Although S.M. uses cues such as clothes and voice to identify individuals, these cues are clearly limited in their efficacy. There has been minimal or no functional change in S.M.'s ability to recognize visual images over the years after a motor vehicle accident (Gauthier, Behrmann, \& Tarr, 1999). In the current study, S.M. was trained over 31 sessions to recognize Greebles at an individual level. While tracking changes in his Greeble recognition, we also measured his performance on nontrained Greebles, faces, and objects (see Figure 2) before and after the intervention and at regular intervals over the course of training. Finally, we obtained pre- (Marotta, Behrmann, \& Genovese, 2001) and postintervention functional magnetic resonance imaging data using these same stimuli. This longitudinal approach allows us to compare experience-dependent changes using behavioral and neuroimaging assays within the same individual.

\section{RESULTS}

The results are divided into 3 sections. We consider first whether S.M. improved in Greeble recognition as a function of the training. We then explore generalization
Figure 2. Examples of the stimuli used in the pre- and posttraining functional imaging studies. Epochs included blocks of 20 gray-scale images of (A) Greebles, (B) Common objects, and (C) Faces.

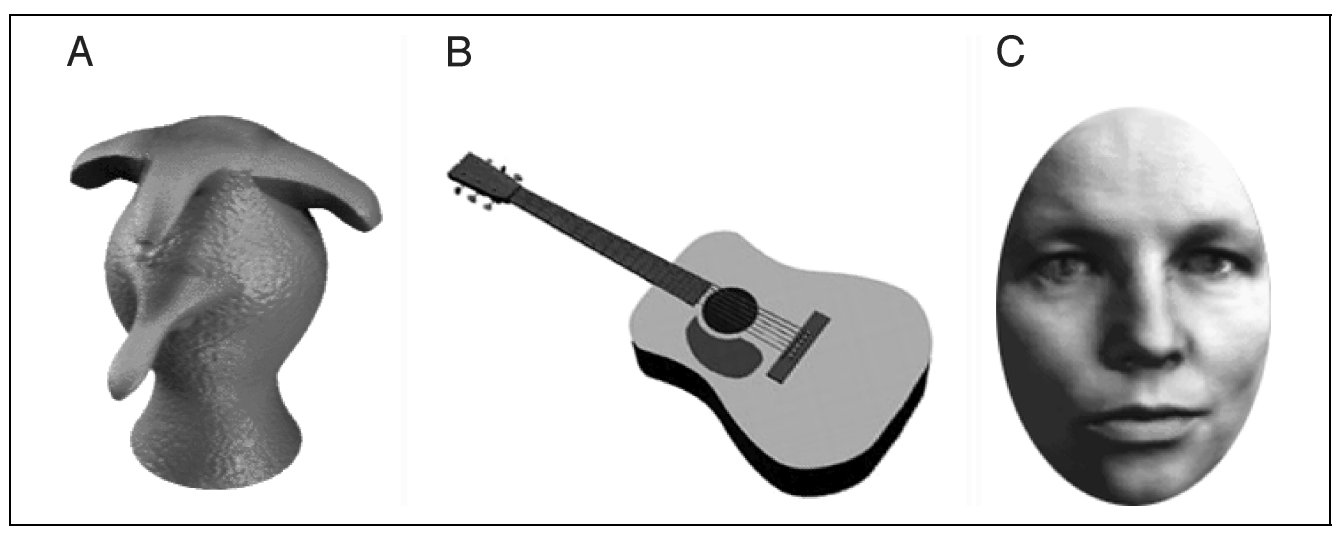


to other visual classes. Finally, we compare the pre- and posttraining imaging data to determine the neural correlates of any behavioral changes.

\section{Greeble Training}

To determine whether S.M. showed any improvement as a function of Greeble training, we compared both his accuracy and RT over the training sessions. Because of the increased chance of spurious results from so many data points, we binned the data from the 31 sessions into 8 time periods ( 4 sessions per time period except the last one, which included 3 sessions). In addition, although only 4 tasks were performed, because the gender individual match task contained both gender and individual match trials in randomized format, we split the data into the 2 subtasks and analyzed each separately. We refer to the blocked data as gender match and individual match and use these same labels preceded by random for the randomized data. An analysis of variance (ANOVA) with time (1-8) and task (gender match, individual match, random gender match, random individual match, and target match) was performed with trial as the random factor. ${ }^{1}$

\section{Accuracy}

S.M. showed significant improvement in accuracy over session, $F(1,6)=12.1, p<.01$, as shown in Figure 3A. Some tasks were clearly more difficult for him than others, $F(4,24)=50.1, p<.0001$, notably those requiring individual level recognition (random individual match and individual match). There was a significant Task $x$ Time Period interaction, $F(4.24)=8.3, p<.001$, reflecting the finding that by Time Period 8, S.M. scored close to ceiling on the easier but not on the more difficult tasks.

\section{Reaction Time}

The improvement in accuracy is mirrored in RT (see Figure 3B). A 3-way ANOVA with Time Period (binned as above), task, and response (yes, no) with correct RT as the dependent measure indicated that all 3 main effects were significant [session: $F(1,6)=33.6, p<.01$; task: $F(4,24)=29.5, p<.0001$; response: $F(1,6)=7.1$, $p<.05]$. There was also a significant Time Period $\times$ Task interaction, $F(4,24)=8.2, p<.01$. Even in Time Period 1, performance on target match and sex match was fast. In contrast, identifying an individual Greeble by verifying its name was considerably more difficult initially, to a slightly greater extent in randomized than blocked trials, and, although S.M. showed considerable improvement, his performance was still not as good as in the other conditions. Of note is that it took S.M. far longer than normal subjects to acquire individual Greeble names

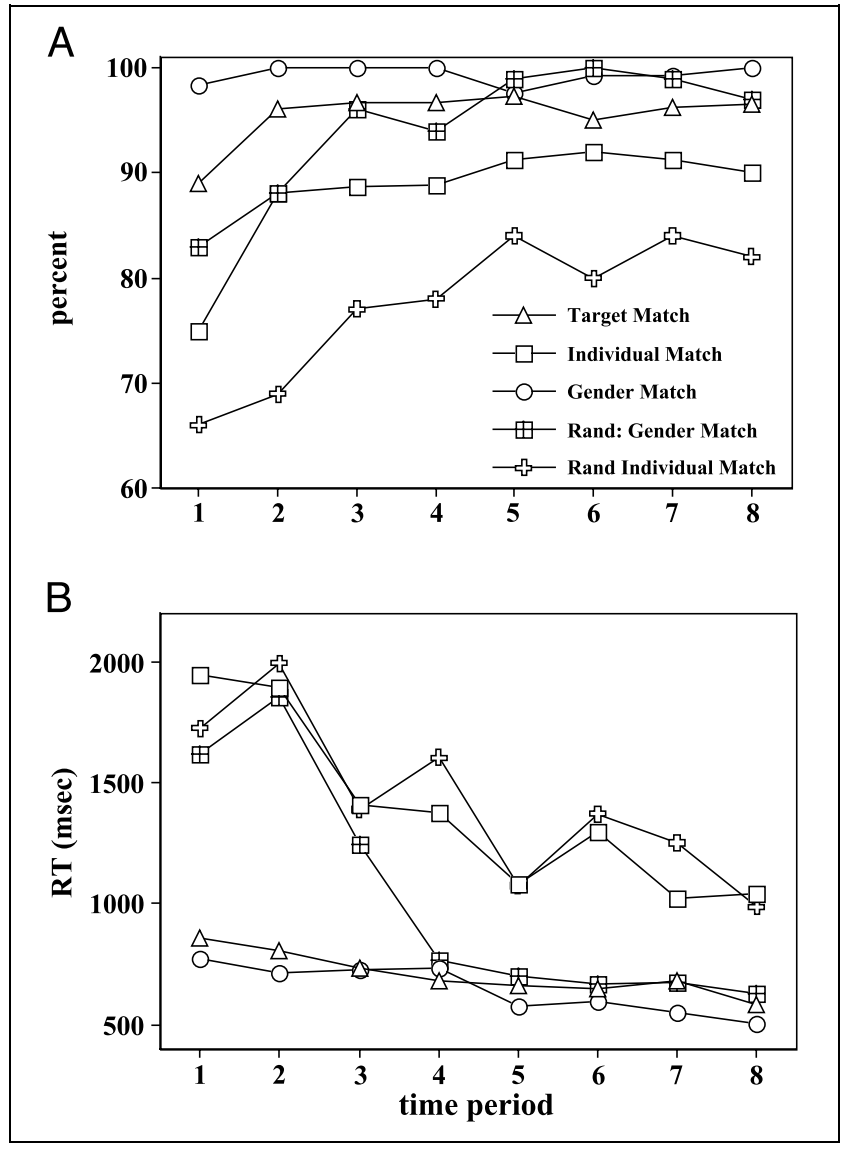

Figure 3. (A) Percent accuracy and (B) Mean RT for S.M. on the Greeble training tasks across 31 sessions of training, binned into 8 time periods.

despite the fact that he only learned to identify 5 Greebles whereas normal subjects can learn 20 Greebles in 7-10 hr of training (Gauthier \& Tarr, 1997).

The critical finding from these analyses is that S.M. benefited positively from training and learned to recognize individual Greebles. In and of itself, this is an important result and augurs well for potential therapeutic interventions for individuals with acquired visual recognition deficits. As is evident, the amount of change differed across the tasks: S.M. improved to a greater extent for harder than for easier tasks, but this is not surprising, given the greater room for improvement in the former case. The claim, then, is not that S.M. learns in the same way that intact individuals do, only that he did benefit positively from this training.

\section{Performance on Generalization Tests}

Having established that S.M. did improve significantly in his Greeble performance, one major issue is the extent to which there is generalization to untrained Greebles and to other classes of stimuli, such as objects and faces. To assess this, we compared his performance on the generalization measures, collected at 5 regular intervals 
over the course of his training. In these tasks, a pair of stimuli appeared on the screen for an unlimited duration and S.M. made a same/different judgment. Both RT and accuracy data were collected for all tasks.

\section{Greebles}

An ANOVA with time period (1-5) and "different" response conditions (basic, family, sex, and individual) on trials using untrained Greebles revealed a significant effect of time period, $F(4,12)=10.8, p<.0001$, with a 1128.5-msec difference between Time Periods 1 and 5 (see Figure 4A, left). Not surprisingly, RTs differed across condition with fastest RT at the basic level (2007.4 msec) and slowest RT at the individual level (4451.3 msec), $F(3,12)=53.1, p<.0001$. There was also a significant Time Period $\times$ Condition interaction, $F(12,572)=2.01$, $p<.05$, reflecting the minimal change across time periods for the basic and sex conditions and the significant change for the family and individual (except for the one anomalous and unexplainable datum for family at time 4). Of note is that the change from Time Periods 1 to 5 for the individual condition is approximately $2 \mathrm{sec}$. This dramatic improvement reveals that the training generalized to recognition of other Greebles. Again, it is worth noting that at the end of training, S.M. still required on the order of $4 \mathrm{sec}$ for individual Greeble judgments, far longer than that required by normal subjects.

Despite the long RTs, S.M. was highly accurate on these untrained Greebles (see Figure 4A, right). A loglinear analysis with time period and conditions, as above, yielded neither an effect of time period nor condition, given that accuracy was at ceiling on this task across all testing sessions $\left(\chi^{2}=0, p=1\right)$. S.M. does not obviously appear to be trading speed and accuracy, and accuracy is high for all Greeble tasks.

\section{Objects}

An ANOVA with time period and "different" conditions (basic, subordinate, exemplar) revealed a significant improvement with a $538.8 \mathrm{msec}$ improvement from Time Period 1 (2267.7 $\mathrm{msec})$ to 5 (1728.9 msec), $F(4,12)=$ $5.03, p<.001$ (see Figure 4B, left, note difference in $y$ axis). There was a difference as a function of condition, $F(2,12)=18.8, p<.0001$, but this was qualified in a Time Period $\times$ Condition interaction, $F(12,447)=$ $2.23, p<.01$, with maximal change from Session 1 to Session 5 (1085 msec) at the exemplar level, $365 \mathrm{msec}$ at the subordinate level and $501 \mathrm{msec}$ at the basic level. As above, S.M. performed more slowly than controls on this task (Gauthier, Behrmann, et al., 1999).

As before, we conducted a loglinear analysis with the factors of condition and time period on the accuracy data. The number of errors for objects was low overall (41 out of 600 ) (see Figure $4 \mathrm{~B}$, right). There were more errors in some conditions than others, $\chi^{2}(3)=64.9$, $p<.0001$, with accuracy lower for both SE and E trials compared with the basic level and to the BSE level, and these 2 latter conditions did not differ from each other. That accuracy does not change over session is not surprising again given the unlimited exposure duration. The RT analyses are clearly more revealing in this case.

\section{Faces}

An ANOVA with time period and "different" conditions (gender, individual) revealed a significant change across time period, $F(4,8)=4.9, p<.004$ (see Figure $4 \mathrm{C}$, left). Unlike the positive change observed in the Greebles and objects generalization, however, the direction of the change was in the opposite direction. S.M.'s performance was worse with faces over time. There was also a significant difference between conditions $F(1,8)=5.01$, $p<.01$, with trials requiring individual discriminations taking longer than trials requiring gender judgments. The condition effect was qualified in an interaction with time period, $F(8,124)=3.3, p<.005$, reflecting the finding that RT for gender trials, was somewhat variable across time periods, but there was significant and incremental slowing across time period for individual trials. The mean RT for Time Period 1 individual trials is $3329.3 \mathrm{msec}$, whereas it is $5567.1 \mathrm{msec}$ for Time Period 5, reflecting a decrement of $2237.6 \mathrm{msec}$.

The loglinear analysis on the accuracy data (see Figure $4 \mathrm{C}$, right) reveals no effect of time period but a significant effect of condition, $\chi^{2}(2)=56.5, p<.0001$, with poorer accuracy for individual than for gender trials. Although not significant, there is a slight trend for accuracy to be decreasing in later sessions compared with the earlier time periods. This is consistent with the claim that performance on face recognition is getting worse.

To confirm that the decrement in face processing does not simply arise from a speed-accuracy trade-off, we calculated $A^{\prime}$, a nonparametric index of sensitivity, across the time periods. ${ }^{2}$ There is no clear correlation between $A^{\prime}$ and RT, suggesting that the slowing in RT in later time periods is not yielding a concomitant improvement in sensitivity; $A^{\prime}$ is roughly equal in individual trials for Time Periods $2,3,4$, and 5 (0.83, 0.89, 0.83, and 0.87 , respectively) and yet there is a large increase in RT across these time periods. It is not the case, then, that S.M. is merely becoming more accurate over time at the expense of slower RTs.

To further confirm the decrement in face processing, we analyzed data from an additional task that S.M. performed only at Time Periods 1 and 5. In this task, 3 faces appeared on a computer screen, a target above, and 2 choices below it on the left and right. S.M. pressed the left or right button to indicate whether the target matched the left or right face. Each face was presented on a square $\left(7.5 \mathrm{~cm}^{2}\right)$. On a single trial, the faces were all upright or all inverted and orientation was blocked. 


\section{A Greebles}
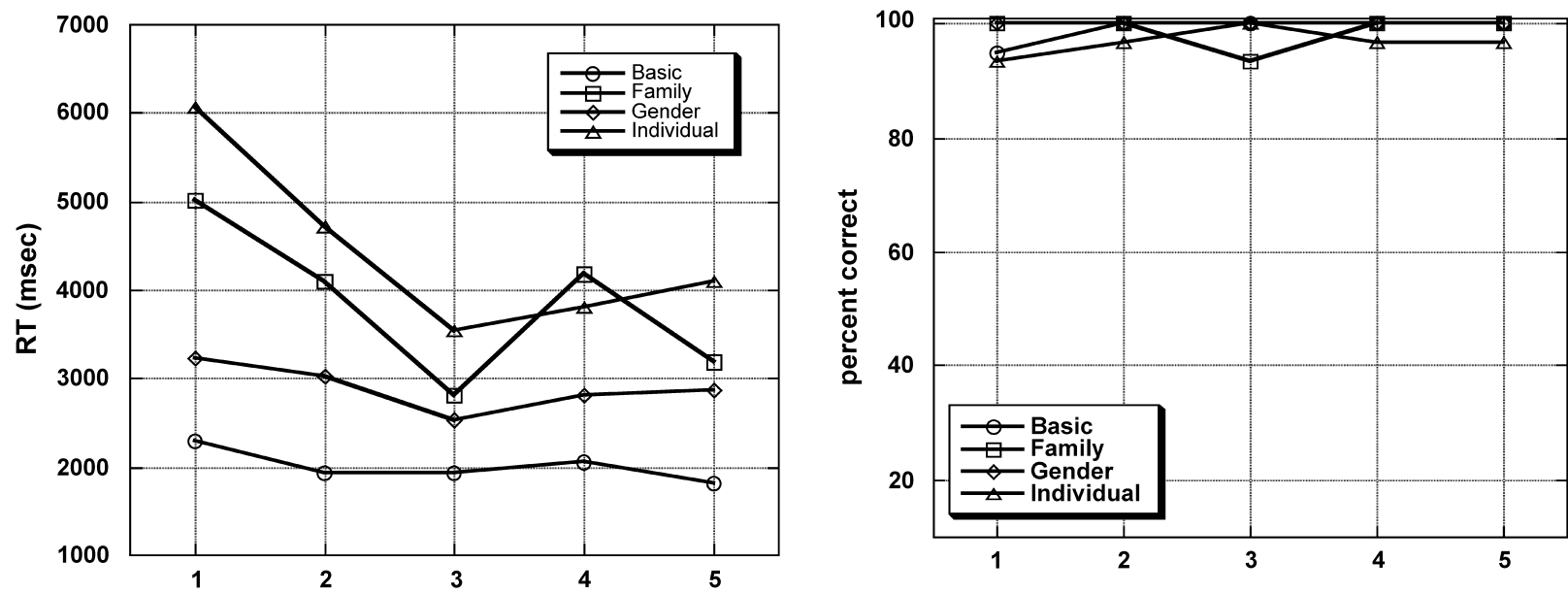

\section{B objects}
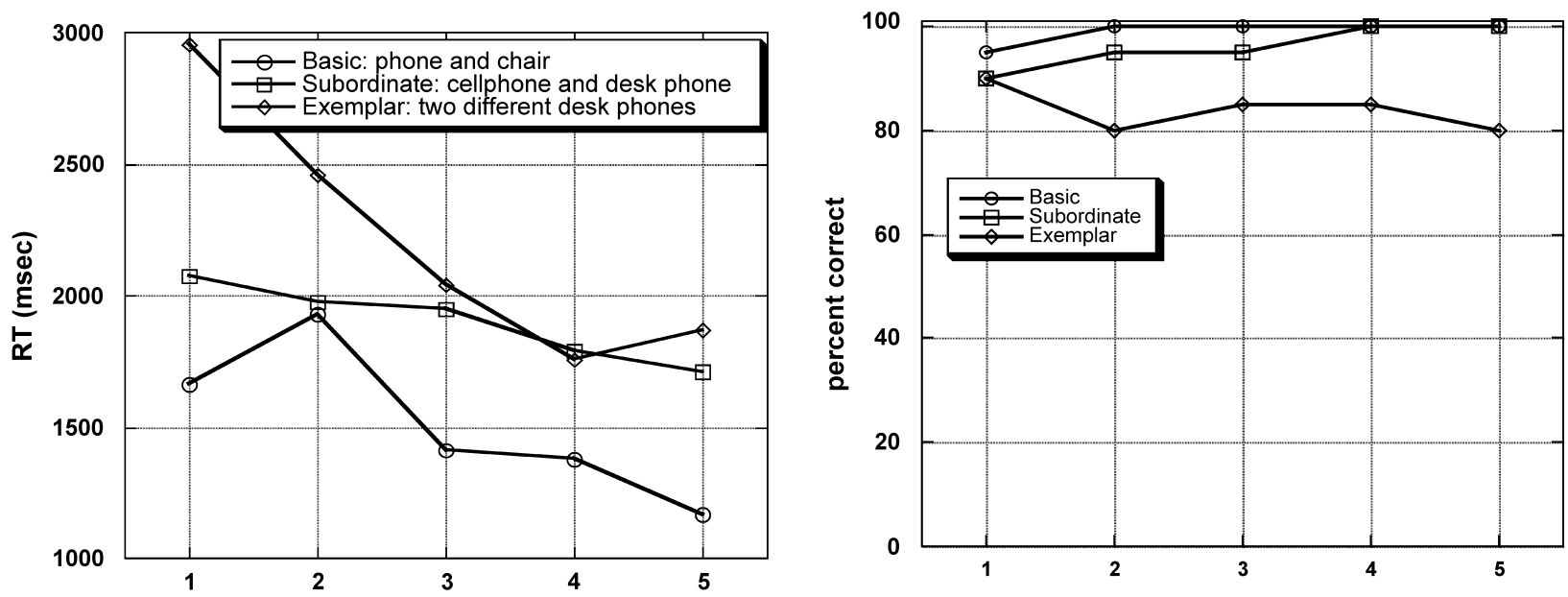

\section{Faces}
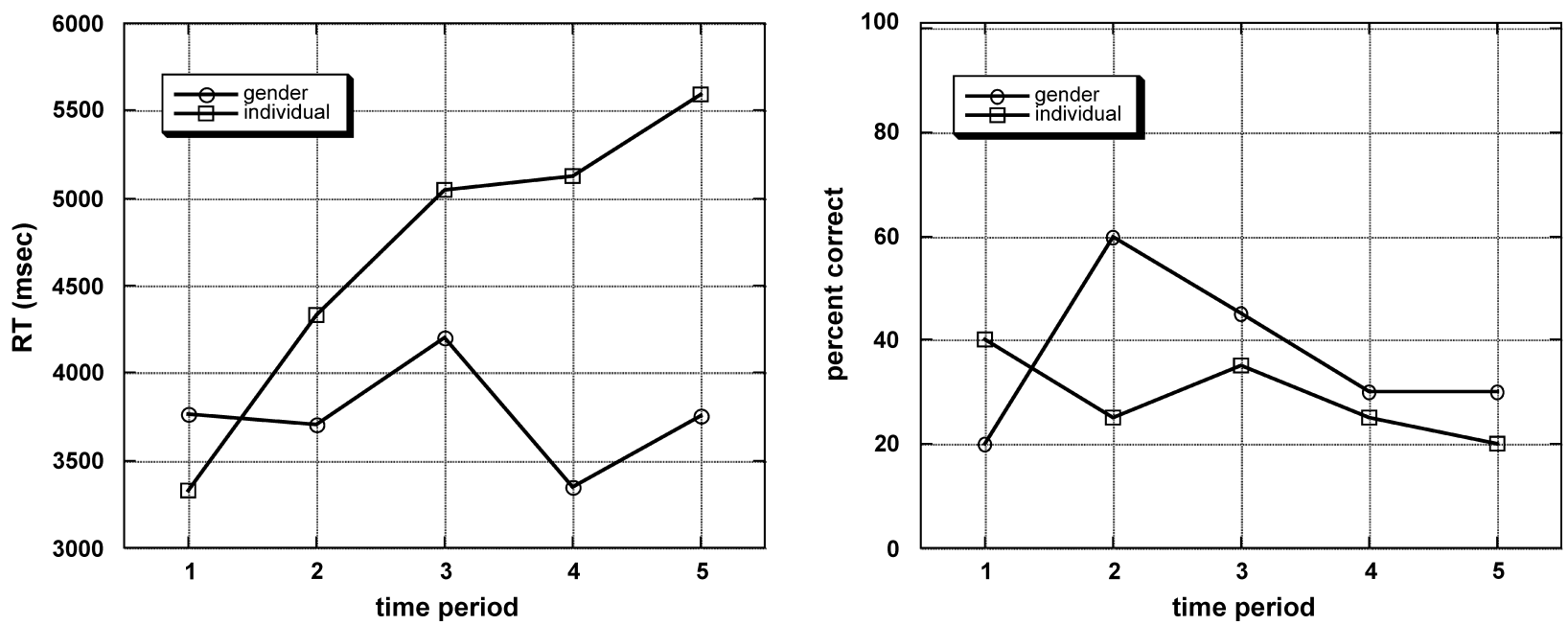

Figure 4. Mean RT (left) and accuracy (right) for S.M. on the generalization tasks as a function of condition over 5 testing sessions for (A) Greebles, (B) Objects, and (C) Faces. 
There were 104 trials of each orientation. The faces were taken from the MPI face database; the stimuli consisted equally of male and female faces and were shown in color. The faces were collected as 3-D models and hair was cropped. S.M. performed the upright trials before the inverted trials on both occasions. Normal subjects are, on average, $300 \mathrm{msec}$ faster for the upright than inverted faces (Marotta et al., 2001). S.M. performed more slowly than the controls but, more importantly, performance was $1373 \mathrm{msec}$ slower in Time Period 5 than $1, F(1,103)=182.8, p<.0001$. Orientation did affect performance, $F(1,103)=61.2, p<.0001$, with a 113-msec advantage for upright over inverted faces but no interaction between time period and orientation, $F<1$. The crucial result is the confirmation of the decrement in performance on faces as reflected in the increase in RT across time periods. Note that the typical advantage for upright over inverted faces is reduced in S.M. relative to controls.

In summary, the findings from the generalization measures are interesting and counterintuitive. The data indicate that S.M. did improve in his processing of novel Greebles that were not part of the training set; because accuracy was generally high at all times but RT was slow, this change manifests in the RT measure. Along with this, S.M. also improved in his processing of common objects, again manifest in RT. These changes are not simply attributable to practice effects over time (given that the stimuli are repeated across 5 time periods) or a generic speed-up in RT as a consequence of visual training, as his face processing is not subject to this same positive change. In dramatic contrast to the Greebles and objects, S.M.'s ability to process faces was negatively impacted as measured in multiple ways and on different paradigms. The decrement in face processing was also not a consequence of a simple speed-accuracy tradeoff. These findings suggest that S.M. benefited from the Greeble training and that there was some positive transfer to object processing. In contrast, his face recognition was adversely impacted. Given the evidence for this adverse change in face processing for S.M., we terminated the training regimen at this point and collected posttraining imaging data.

\section{Pretraining and Posttraining Imaging Comparison}

To analyze the imaging data, we first placed a mask over the entire extent of the fusiform gyrus and then set a threshold for identifying significantly activated voxels in the comparisons of interest. Because the 3-T scanner, which was used for pretraining imaging was being upgraded at the posttest time, we acquired S.M.'s posttraining data on the 1.5-T scanner and collected control data on that scanner as well. To identify thresholds for the individual subtractions and to take into account a potential difference in absolute activation given the different scanners, we used the FDR procedure for multiple testing under dependent tests (see Methods). Then, we calculated the number of activated voxels in the fusiform ROI that reached this threshold in the subtraction of interest and performed all pairwise subtractions of faces, objects, and Greebles (faces - Greebles, objects - Greebles, faces - objects). We also examined performance in 2 additional conditions: faces - fixation and Greebles - fixation to evaluate any preto posttraining changes against the baseline.

An omnibus ANOVA with group (S.M., control), scanner $(3 \mathrm{~T}, 1.5$ that delineates pre- and posttraining for S.M.), hemisphere (left, right), and subtractions (faces Greebles, objects - Greebles, faces - objects) was performed. The dependent measure was the number of activated voxels, measured along 5 different points from posterior to anterior along the fusiform gyrus. We normalized this measure by the total number of activated voxels to account for differences in extent of activation across individuals. The critical finding is a 3-way interaction among scanner, subtraction, and group, $F(2,20)=3.6, p<.005$. The 4-way interaction of these variables with hemisphere did not reach significance, $F(2,20)=2.8, p=.08$, and so the data are collapsed across hemisphere for the purpose of discussion. Figure 5 reflects the number of voxels activated for the various subtractions for the (A) control subjects and (B) S.M. on the 3- and 1.5-T scanners, which refer to preand posttraining acquisition for S.M. Post hoc comparisons were done with $p<.003$, corrected for multiple comparisons. To understand these interactions, we separated out the controls and S.M. for further analysis and discussion.

\section{Control Subjects}

As is evident from Figure 5A, for the faces - Greebles, faces - objects, faces - fixation, and Greebles - fixation subtractions, there is no statistically significant difference in the number of activated voxels for the 3 - and 1.5 -T scanner $(F<1)$. There is, however, greater activation for Greebles than for objects, as indicated by the negative values. This particular pattern has been observed previously (Gauthier, Tarr, et al., 1999) and is attributed to the greater homogeneity of the Greeble requiring more fine-grained discrimination than in the case of object stimuli. This difference is greater for the 3than 1.5-T activation, perhaps reflecting greater sensitivity in the former than latter, but it is against this pattern of data that we examine any changes in S.M.'s behavior over time.

\section{S.M.}

The major result from the imaging data is that we see differences between the pre- versus posttraining (3- vs. 1.5-T) activation for S.M. in each of the comparisons 
Figure 5. (A) Mean number of (normalized) activated voxels for control subjects in 5 different subtractions on the 3- and 1.5-T scanner and (B) number of (normalized) activated voxels for S.M. in 5 different subtractions on the 3-T (pretraining) and $1.5-\mathrm{T}$ (posttraining) scanner.

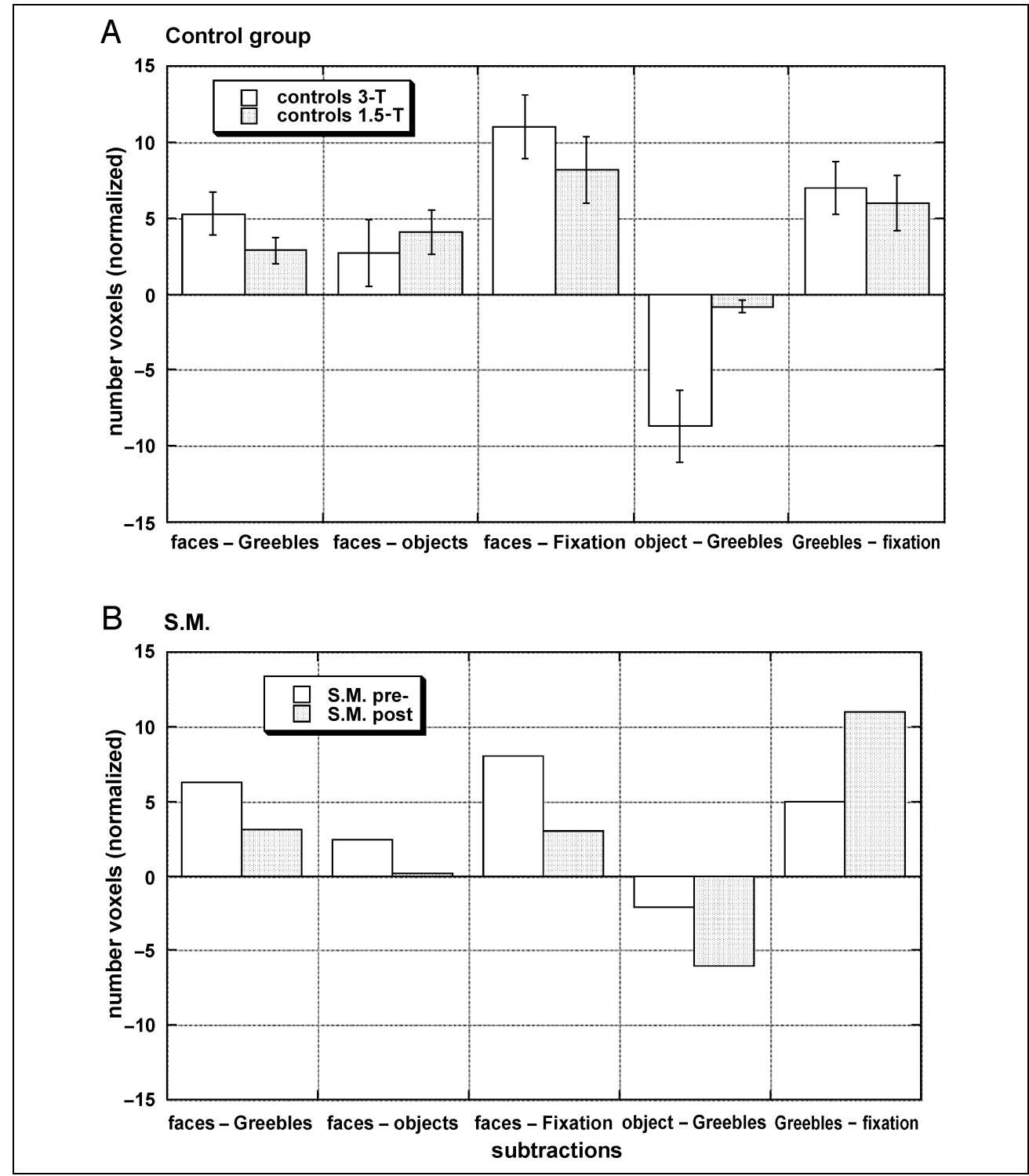

$\left(\chi^{2}, p<.05\right)$, as is evident in Figure 5B. In the faces - Greebles comparison, there were significantly more voxels (6.5) activated prior to intervention than at termination of training (3.5). Although this suggests a decrease in face selectivity, this result in and of itself is not definitive as the reduction in activation from 3 to $1.5 \mathrm{~T}$ for the control subjects is of approximately the same magnitude (cf. Figure 5A,B). The reduction in face selectivity, however, is confirmed in the faces - objects subtraction in which there were 2.5 voxels activated initially and only 0.2 activated at the end of the training time period. These findings parallel the behavioral data in which we see a decrement in face processing at the end compared with the beginning of training. To explore this neural correlate further, we examined the faces - fixation subtraction for S.M. and similarly found a reduction in face selectivity even in this subtraction. This reduction in face selectivity can be seen in
Figure $6 \mathrm{~A}-\mathrm{C}$, which shows a single slice from the preand posttraining functional images in which there is considerably reduced posttraining activation for faces even in comparison with a fixation baseline. Prior to training, depending on the subtraction, a more posterior right fusiform and a more anterior left fusiform face site are observable. These sites are not surprising, given S.M.'s right-sided lesion and the possible pretraining recruitment of left hemisphere substrate for face representation following right fusiform damage (Marotta et al., 2001). Interestingly, both of these sites show a reduction in activated voxels posttraining.

The decrement in face-selective voxels observed above might come about not only because of the reduction in face selectivity but also because of an increase in Greeble and object selectivity, and so we examine these particular patterns next. In the object Greebles comparison, there is some, albeit minimal 
Figure 6. Functional activation patterns represented on an axial slice in S.M. pre- and posttraining imaging sessions in the ROI for the (A) faces Greebles subtraction, (B) faces - object subtraction, (C) faces - fixation subtraction, (D) objects Greebles subtraction, and (E) Greebles - fixation subtraction. Following radiological convention, the right hemisphere appears on the left of the display.

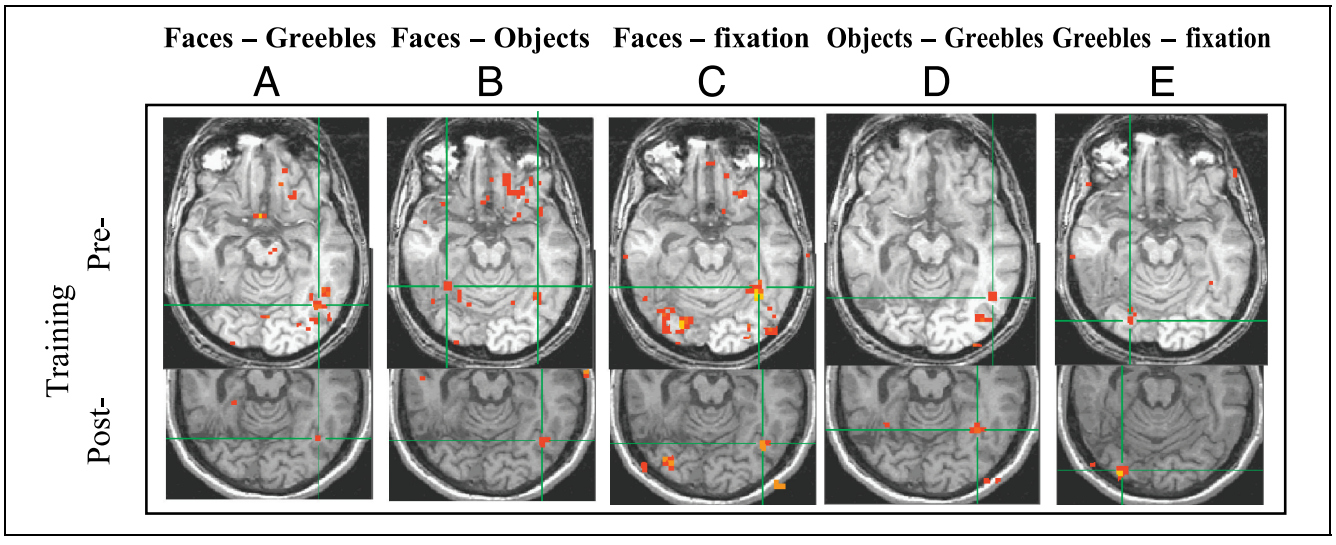

Greeble-specific activation pretraining (as reflected in the negative value in Figure 5B) but there is an increase in the number of voxels activated by Greebles compared with objects posttraining. The behavioral improvement on the Greeble tasks posttraining is therefore supported by the increment in the number of Greeble-selective voxels. It is also the case that a subtraction of Greebles against fixation, as shown in Figures 5B and 6E, reveals the increase in Greeble selectivity.

Taken together, the neuroimaging data reveal significant changes in S.M.'s pattern of BOLD activation prior to versus postintervention. Not only does he show an increase in the number of voxels that are responsive to Greebles posttraining, but he shows a concomitant reduction in face-selective voxels. Note that the apparent reduction in activated voxels for the faces - Greebles, faces - objects, and faces - fixation subtractions cannot be attributed to the shift from a 3- to 1.5-T scanner, as the object - Greebles and Greebles fixation subtraction yields the reverse finding. In addition, a comparison of the data from the normal subjects does not show a significant difference in favor of the 3-T scanner. In spite of the fact that the comparison between the control subjects and S.M. is statistically complicated (pitting a group against a single subject) and that single-subject imaging data are notoriously variable, we obtain robust evidence for a neural correlate of the behavioral changes in S.M.'s performance.

\section{DISCUSSION}

This study documented the behavioral and neural changes in S.M., an individual with relatively longstanding visual object agnosia and prosopagnosia, following a focused visual retraining program. The first goal of the study was to examine S.M.'s potential for improvement in recognizing a set of novel visual stimuli-Greebles-after intensive training and to examine the neural substrate mediating any behavioral change. The second goal was to examine the extent to which Greeble training gen- eralized to other categories of visual objects, including untrained Greebles, common objects and faces to shed light on the shared mechanisms underlying recognition of a variety of visual stimuli (faces, objects, Greebles).

\section{Experience-dependent Change in Prosopagnosia}

To evaluate the potential for learning, we implemented a 31-week training program in which S.M. learned to make relatively simple sex (appendages pointing up or down?) judgments and to make individual judgments, assigning an individual identity to each specific Greeble. We adopted this training regimen both because it has been shown to be successful with normal subjects in inducing behavioral effects typically associated with face processing and expertise, and because posttraining neuroimaging data has revealed recruitment of the fusiform gyrus, the very area that mediates some aspects of face processing.

The first major result was that S.M. was indeed able to learn to discriminate between Greebles at a coarse level (gender) and, importantly, at a more specific level of categorization (assigning a label to an individual Greeble). Although the acquisition of these abilities was far from normal, taking S.M. roughly 4 months of training with 5 individual Greebles (when normal subjects learn to identify 20 individual Greebles in 7-10 hr), S.M. did exhibit significant improvement in Greeble recognition. In addition, tested on a set of untrained Greebles, S.M. showed improvement in discriminating among them, reflecting generalization, a pattern also observed in normal subjects (Gauthier \& Tarr, 1997).

The behavioral improvement in Greeble recognition was mirrored in the neuroimaging data; a significant increase in posttraining suprathreshold voxels was observed for Greebles compared with faces, with other common objects, and in comparison with a fixation condition. That relearning and concomitant dynamic cortical reorganization is possible in an adult with longstanding agnosia who has not received any form of 
intervention, aside from the therapy immediately after the brain damage, is interesting and exciting in and of itself (for related work in dyslexia, see Temple et al., 2003). We should note, however, that not all patients benefit from such intervention. A procedure similar to the one adopted here was also used by Caldara et al. (submitted) who trained a prosopagnosic patient, P.S., to recognize individual Greebles. Note that P.S. has a "normal" fusiform face area on the right, despite the apparent absence of feedforward inputs from the right occipital area (Rossion et al., 2003). Despite training that extended 3 times as long as for the normal controls, P.S. did not learn to recognize Greebles. In addition, a third prosopagnosic patient, L.R., trained by Bukach et al. (in preparation) learned to identify 20 Greebles at the individual level and ultimately reached criterion for expertise. However, as with S.M., L.R.'s performance did not appear "normal": She performed more poorly than normal individuals for unknown Greebles, had slower and more variable responses, and took a greater number of sessions to reach criterion. In light of these differences, it behooves us, in future, to explore under what conditions this type of intervention approach is beneficial and which candidates are most likely to benefit from this intervention. The findings from the current study, however, are the basis for some optimism, revealing that an agnosic patient can be trained to perform visual object recognition on novel objects even several years after brain damage had been sustained.

An immediate question concerns the neural mechanisms that might be implicated in this experiencedependent change. This issue is of much current interest and several single unit recording studies in awake, behaving monkeys have documented the capacity of single neurons in inferotemporal cortex, the region that potentially mediates the change in S.M., to be sensitive to change. For example, the configural sensitivity revealed by some temporal neurons (Perrett \& Oram, 1993) may be enhanced by training and can be finetuned to the parameters and statistics of the input (Hasegawa \& Miyashita, 2002). The change in neural selectivity that follows training is also well illustrated in a recent study in which neurons in monkey inferotemporal cortex exhibited greater neural selectivity in response to a trained conjunction of a top and bottom parts on an object than to either the top or the bottom part presented alone (Baker, Behrmann, \& Olson, 2002). This sensitivity was much more evident, however, for trained (highly familiar) combinations than for untrained (less familiar) conjunctions, implicating familiarity and expertise as relevant factors.

\section{Implications for Organization of Ventral Visual Cortex}

The second major result was that S.M. showed some generalization from the training, exhibiting improved processing of nontrained Greebles and of other common objects. Most striking however, S.M. showed a decrement in his ability to recognize faces posttraining compared with pretraining. In fact, once this became definitive, we terminated the training program. The deterioration in his face processing, as revealed on the generalization task, was not attributable to a speed-accuracy trade-off and was also observed using multiple paradigms. The decline in his face recognition ability was mirrored in the neuroimaging data in 3 different ways. Whereas there were more voxels activated for faces than Greebles at the start of the intervention, significantly fewer face-selective voxels were identified posttraining. The same pattern was evident in the comparison between faces and objects with a decrease in activated face-selective voxels posttraining compared with pretraining. Finally, even when faces were compared with fixation, we see a reduction in the number of activated voxels, attesting to the dramatic drop-off of face-specific activation.

Of particular interest is the apparent competition between faces and Greebles: As Greeble recognition improves, there is a concomitant decrement in face recognition. One obvious explanation for this relationship is that the psychological and/or neural systems that mediate face and Greeble recognition is shared and that when these systems are fine-tuned to the properties of Greebles through training, so they are tuned away from the details of faces. The implication of this is that although both classes may require configural processing, there still remain differences between these visual classes (Gauthier, Behrmann, \& Tarr, 2004). It is also possible, however, that in individuals who have not sustained brain damage, there is sufficient neural tissue to be able to represent both stimulus classes without cost (and there is no obvious cost to face processing in individuals who were trained as Greeble experts). In the case of S.M., however, who does not have a full complement of inferotemporal neurons, neural capacity is limited and the residual tissue becomes dynamically sensitized to the major task at hand. The frequent exposure to Greebles and the pressure to attain individual recognition works against his remaining ability to recognize faces. We should note, however, that interference effects may be observed with some normal subjects too under very particular conditions. In a recent study, Gauthier, Curran, Curby, and Collins (2003) demonstrated that holistic processing of faces (a supposed hallmark of the face module) is interfered with by holistic processing of cars in car experts, indicating that the 2 are not functionally independent. Moreover, the extent of the interference increased with greater expertise in car expertise. Event-related potentials suggested that this interference arose at a fairly early stage of processing, likely implicating holistic processing for faces and cars. Thus, in individuals who have normal visual recognition but in whom the neural tissue is specifically co-opted for car discrimination, there is a subsequent cost for face 
recognition, just as in S.M.'s case, and this cost occurs at the level of configural processing.

The argument we have presented so far suggests a common neural substrate for face and Greeble recognition, with competition for representation in this shared substrate. There are, however, alternative, although not mutually exclusive explanations that also deserve consideration. In addition to the improvement in Greebles and the reduction in face recognition, S.M. also exhibited a slight improvement in common object processing following Greeble training. The improvement for objects might be attributed to an expansion in his partbased (rather than configural-based) processing. Many studies have recognized the failure of individuals with prosopagnosia following a fusiform lesion to perform configural-based tasks. For example, Barton, Press, Keenan, and O'Connor (2002) showed that 4 such patients were unable to discriminate changes in the spatial position of face components but were able to detect changes in the color of the components (e.g., the eyes). Because S.M. has a lesion to this very area, he may be unable to reacquire the ability to perform configural computation. This might explain why he did succeed in Greeble training but not to the same extent as normal individuals. Furthermore, the increase in part-based processing is likely to be particularly detrimental for face processing, which depends heavily on configural representations. Whereas Greeble training may be beneficial for individuals with intact fusiform gyri, the training was not as advantageous for S.M. and, although he did acquire some visual skill, it was not without cost.

A further issue that may be relevant for the observed Greebles-faces interference concerns the nature of the training. The Greeble training was intensively conducted across the 31 sessions and no faces were trained during this time period. Although the face testing was conducted (to assess generalization) at the regular 5 intervals and S.M. clearly was exposed to faces during the course of the weeks of Greeble training, the restriction of training to Greebles constituted massed practice. Interestingly, we know that neural networks trained using massed practice and without interleaved trials of the different stimuli (akin to Greebles and faces here) show catastrophic interference, whereas networks trained with interleaved trials are not subjects to this interference (McClelland, McNaughton, \& O'Reilly, 1995). The presence of this interference should serve as a cautionary note to investigators interested in replicating this form of training. An interleaved form of retraining might mitigate some of these effects and offset the catastrophic interference.

We have no way of adjudicating between these alternative explanations of Greeble-face interference at this stage and clearly further research is necessary to understand better the mechanism of change and the candidate individuals who would benefit from such change. At the same time, we feel that studies such as this, which address not only the impairments of individuals with brain injury, but also their intact or reacquired abilities, are essential in gaining a better understanding of neuropsychological case studies and their implications for larger questions within cognitive neuroscience.

\section{METHODS}

\section{Case History}

S.M., a right-handed, 24-year-old English-speaking man, consented to participate in this study. At the age of 18 years, S.M. sustained a closed head injury in a motor vehicle accident. Despite extensive injuries, he recovered well after rehabilitation, aside from the persisting visual agnosia and prosopagnosia. CT scans obtained during the initial trauma period indicated a contusion in the right anterior and posterior temporal regions accompanied by deep shearing injury in the corpus callosum and left basal ganglia. At present, S.M. has resumed most aspects of normal life. He has enrolled in a community college, taking courses in communication (although he requires assistance with the visual material), and works in his family's store.

S.M. has corrected visual acuity to $20 / 20$ and his vision is unremarkable in all other respects. He performs within the normal range on tests of low-level visual processing (judging size, length, orientation of stimuli, color, and motion) as well as on tests that require matching of objects from different viewpoints or along a foreshortened axis (Riddoch \& Humphreys, 1993). S.M.'s accuracy in identifying the line drawings of the Boston Naming Test was 66\% (171/260; normal 96.4\%) and his mean reaction time (RT) was 2.14 sec per image (normal $884.67 \mathrm{msec}$ ). S.M. is also disproportionately slowed, relative to normal subjects, in discriminating between exemplars within a single category (e.g., snowflakes, Greebles, or houses), and the impairment is magnified as the perceptual similarity between the stimuli increases. His prosopagnosia is also profound: When shown 40 black-and-white photographs of famous individuals (e.g., Bill Clinton or Marilyn Monroe), he was unable to recognize any, despite being able to provide a good verbal identification when presented with their name auditorily. S.M. performs in the "severely impaired" range (36/54 and 32/54 on 2 separate occasions; normal 41-54) on a standardized test of facial discrimination, the Benton Faces test. S.M. has been described previously and the reader is referred to those articles for further information (Behrmann \& Kimchi, 2003; Marotta et al., 2001; Gauthier, Behrmann, et al., 1999).

\section{Training Procedure}

\section{Stimuli}

Stimuli for this experiment consisted of novel objects, Greebles, rendered from 3-D models with Alias Sketch 
software (Alias Research, Toronto). All Greebles have 4 protruding parts organized in approximately the same spatial configuration along a vertically oriented central "body" part. The entire set of Greebles is organized orthogonally along 2 categorical dimensions, with each Greeble being a member of 5 possible "families," defined by the central body shape, and of 2 possible "genders," defined by the orientation of the parts relative to the central part, either all pointing upward or downward. Although some of the parts are very similar to each other, every individual part is unique within the set. Thirty Greebles (three individuals from each gender by family combination) were used during training (see Figure 1).

\section{Apparatus and Materials}

The training was conducted on a Macintosh IIci computer using a 13-in. $(33.92 \mathrm{~cm})$ color monitor and RSVP experimental software version 4.0.5. S.M. completed the training at home after receiving detailed instruction and guidance from the experimenters. We collected accuracy and RT data in each session and met with him approximately every 5 sessions to measure his progress on the training set and to examine any transfer to other visual domains (see below).

\section{Design and Procedure}

In this training regimen, S.M. was trained to differentiate gender (parts up or down) and to identify 5 individual Greebles. In each training session, he completed 4 blocks of trials (31 training sessions, at least 2 sessions per week):

(a) Gender match: In each trial, the auditory presentation via sound file of a Greeble gender label (male, female) was followed immediately by the visual presentation of a Greeble, which remained on the screen until response. S.M. was required to respond with a key press, yes or no, if the visual display Greeble matched the gender label. He was trained with the male/female label before initiation of the training procedure ( $n=50$ trials).

(b) Individual match: Trials were identical to gender MATCH trials but the auditory stimulus was the label of the individual identity of a Greeble. S.M. was required to decide whether the label matched the individual Greeble and to respond yes or no with a key press. As above, he was familiarized with the possible names and the Greebles before the beginning of treatment ( $n=50$ trials).

(c) Gender/individual match: To provide further opportunity for training, gender match and individual match trials, described above, were randomized in a block. This was meant to be slightly more challenging for S.M. and is the task used to assess criterion in normal subjects in previous Greeble training regimens. The data from the 2 types of trials are analyzed separately ( $n=50$ each type).

(d) Target match: Trials involved a target Greeble, which appeared inside a big circle accompanied by the auditory label "target." S.M. was required to view this target for roughly $4 \mathrm{sec}$. Following this, a series of 10 Greebles were shown one at a time in random order and S.M. indicated yes or no whether the present stimulus matched the target or not. Trials contained images that were either identical (Ident) to the target or different. When different, trials could include a Greeble from the same family and gender as the target, a Greeble of the same gender as the target but from a different family (body shape), or a Greeble that differs from the target along all 3 dimensions. This task was designed to focus on the different levels of categorization and to encourage S.M. to make increasingly finer discriminations of the individual Greebles $(n=40$ trials $)$.

\section{Generalization Testing Procedure}

At 5 different time points, spaced evenly over the course of the training sessions, S.M. completed the generalization tasks. These were designed to track his performance on nontrained Greebles and to assess any concomitant changes in face and object recognition. On each trial, 2 stimuli (of varying levels of similarity) appeared side by side on a computer screen for an unlimited duration and S.M. was required to decide whether the stimuli were the same or different using a key press. Both accuracy and RT were measured. The trials were randomized across conditions within a block.

\section{Greeble Generalization}

The stimuli consisted of 30 Greebles, none of which were part of the training regimen. Five conditions were tested. The identical condition consisted of 2 identical Greebles, requiring a "same" response. The level of categorization was manipulated across the remaining 4 different conditions ( $n=30$ each), with a Greeble paired with (1) a familiar object, such as a car (basic), (2) a Greeble from another gender but from the same family (gender), (3) a Greeble of different family but the same gender (family), and (4) a Greeble with different individual identity but from the same family and gender (individual).

\section{Object Generalization}

Gray-scale objects $(n=100)$ were obtained from multiple sources, including public domain sites and commercial CD-ROMs. Two stimuli were paired in 5 conditions: (1) a "same" response for identical trials 
(40 trials). A "different" response was given to the following conditions: (2) basic: stimuli differ at basic, subordinate, and exemplar levels (20 trials; e.g., phone and chair), (3) subordinate: stimuli differ at subordinate, and exemplar levels (20 trials; e.g., a digital phone and an old analog phone), and (4) exemplar: stimuli are different exemplars but from the same subordinate level description (20 trials; e.g., 2 different digital phones).

\section{Face Benchmark}

The stimuli consisted of 60 gray-scale faces (half male) scanned from a 3-D laser (obtained from Max Planck Institute, Tübingen, Germany). All faces were cropped using the same $2.25 \times 3$-in. oval window to remove cues from the hairline and face contour. No diagnostic or salient cues are present on these faces. Two faces were paired in 3 conditions with 20 trials per condition. "Same" responses were given to identical trials. "Different" responses were as follows: (1) different gender and (2) different individual.

\section{Imaging Procedure}

Two imaging sessions were conducted at the University of Pittsburgh Magnetic Resonance Imaging Center, the first 2 weeks prior to intervention and the second 2 weeks following the termination of the intervention. Pretraining scanning was done on a 3-T scanner. Because this scanner was being upgraded when we needed to scan posttraining, we acquired the posttraining scans on a 1.5-T machine at the same location. In light of this difference, using the identical paradigms and parameters as for S.M., we tested 2 control groups ( $n=4$ each, age and education matched) on each scanner. Comparisons across the 2 control groups provide information on differences in signal-to-noise ratio on the 2 scanners and serve as a baseline against which to compare S.M.'s BOLD activation at the 2 different time points. Additional measures were undertaken to ensure that any differences pre- versus posttraining for S.M. were not merely an artifact of the different scanners (see Results).

\section{Stimuli}

The stimuli consisted of the same gray-scale images of faces, Greebles, and common objects (Figure 2) used in the generalization tasks. We also obtained scans under 2 additional conditions: where only a fixation point was present or with displays containing scrambled fragments of faces, objects, and Greebles.

\section{Procedure}

The visual stimuli were presented in blocks of $30 \mathrm{sec}$ (20 stimuli per epoch at $1.5 \mathrm{sec}$ per image) and each epoch was repeated 5 times. Subjects were instructed to fixate and to press a key when a circle appeared around one of the stimuli. Circles were drawn around $10 \%$ of the stimuli. S.M. made no errors on this task.

\section{Data Acquisition}

Functional imaging was performed on 3.0- and 1.5-T Signa whole-body scanners (General Electric Medical Systems, Milwaukee, WI) with resonant gradient-echo planar capabilities. Fourteen 3-mm-thick axial planes skip $1 \mathrm{~mm}$ (voxel size $3 \times 3 \times 3 \mathrm{~mm}$ ) were acquired while subjects viewed the stimuli (Time 1 on the 3-T magnet: $\mathrm{TR}=3.0 \mathrm{sec}, \mathrm{TE}=25 \mathrm{msec}$, single shot, matrix size $=128 \times 64 ;$ Time 2 on the 1.5-T magnet: $\mathrm{TR}=$ $3.0 \mathrm{sec}, \mathrm{TE}=50 \mathrm{msec}$, matrix size $=64 \times 64)$. The matrix size for time 1 was clipped to $64 \times 64$ during analysis. Subjects were positioned within a head coil, and head motion was minimized with firm cushions. All stimuli were projected onto a rear projection screen, viewed from an angled mirror fixed to the head coil.

\section{Statistical Analysis}

We compared S.M.'s pre- and posttraining imaging against each other and against the control subjects' data after mapping the anatomical images into Talairach coordinates and then superimposing the functional data. To investigate the patterns of activation, we defined a region of interest (ROI) in both hemispheres that covered the entire fusiform gyrus and examined the distribution of activated voxels along the entire length of this ROI. We then compared the magnitude of relative activation (normalized within subject) across conditions within a session and across the 2 different points in time. To compare the within- and between-session activation patterns for S.M. and the controls, we used a method that controls the false discovery rate (FDR; Genovese, Lazar, \& Nichols, 2002; Benjamini \& Hochberg, 1995) to select a threshold for the voxelwise $t$ statistics that accounts for multiple statistical testing. FDR is the expected proportion of rejected tests that are false discoveries. Methods that control this rate guarantee that the FDR will be below a target level $q$ on average. We used $q=0.01$ along with an adjustment to the method that makes it applicable to arbitrary dependent tests. FDR-based methods have been shown to be more powerful than other available approaches to multiple statistical testing (Marotta et al., 2001). FDR is also especially useful given that we collected data on 2 different scanners, as it adjusts the subtractions to control the FDR on each set of data separately and, thereby, is tailored to the output of the individual scanner.

\section{Acknowledgments}

This research was supported by funding to M.B. from the National Institutes of Mental Health (MH54246 and MH54766); 
to M.B., I.G., and M.J.T. from the James S. McDonnell Foundation (Perceptual Expertise Network 15573-S6), the National Science Foundation (BCS-0094491), and by the McDonnell-Pew program in Cognitive Neuroscience; as well as the National Science and Engineering Research Council of Canada to J.M. We thank Heinrich Bülthoff and Nicolas Troje for access to the Max-Planck Face Database, S.M. and his family for their continued enthusiasm and participation in this research, and finally, Dwight Kravitz and Galia Avidan for their comments and Chris Genovese for discussion of some of the statistical issues.

Reprint request should be sent to Marlene Behrmann, Department of Psychology, Carnegie Mellon University, Pittsburgh, PA 15213-3890, or via e-mail: behrmann@cnbc.cmu.edu.

\section{Notes}

1. We performed the same analyses using all 31 sessions as different levels of the factor and, without binning, the sessions and the same main effects and interactions were obtained. 2. $A^{\prime}$ provides an approximation of the area under the isosensitivity curve. Chance performance yields a score of 0.5 , and more positive values indicate better than chance performance.

\section{REFERENCES}

Baker, C., Behrmann, M., \& Olson, C. (2002). Impact of visual discrimination training on the representation of parts and wholes in monkey inferotemporal cortex. Nature Neuroscience, 5, 1210-1216.

Barton, J. J. S., Press, D. Z., Keenan, J. P., \& O'Connor, M. (2002). Lesions of the fusiform face area impair perception of facial configuration in prosopagnosia. Neurology, 58, 71-78.

Behrmann, M., \& Kimchi, R. (2003). What does visual agnosia tell us about perceptual organization and its relationship to object perception? Journal of Experimental Psychology: Human Perception and Performance, 29, 19-42.

Benjamini, Y., \& Hochberg, Y. (1995). Controlling the false discovery rate: A practical and powerful approach to multiple testing. Journal of the Royal Statistical Society, B57, 289-300.

Bentin, S., \& Deouell, L. (2000). Structural encoding and identification in face processing: ERP evidence for separate mechanisms. Cognitive Neuropsychology, 17, 35-54.

Bosley, T. M., Dann, R., Silver, F. L., Alavi, A., Kushner, M., Chawluk, J. B., Savino, P. J., Sergott, R. C., Schatz, N. J., \& Reivich, M. (1987). Recovery of vision after ischemic lesions: Positron emission tomography. Annals of Neurology, 21, 444-450.

Braus, D. F., Hirsch, J., Hennerici, M. G., Henn, F. A., \& Gass, A. (1999). Brain plasticity after acute encephalomyelitis-A combined structural and functional MRI study of the visual system. Neuroimage, 6, S718.

Burns, M. S. (2004). Clinical management of agnosia. Topics in Stroke Rehabilitation, 11, 1-9.

Buxbaum, L. J., Glosser, G., \& Coslett, H. B. (1999). Impaired face and word recognition without object agnosia. Neuropsychologia, 37, 41-50.

Caldara, R., Gauthier, I., Rossion, B., Schuller, A. M., Tarr, M., \& Mayer, E. (submitted). Prosopagnosia as an inability to develop expertise with visually homogeneous categories: Evidence from a case study.

Diamond, R., \& Carey, S. (1986). Why faces are and are not special: An effect of expertise. Journal of Experimental Psychology: General, 115, 107-117.
Elbert, T., \& Rockstroh, B. (2004). Reorganization of human cerebral cortex: The range of changes following use and injury. Neuroscientist, 10, 129-141.

Farah, M. J. (1990). Visual agnosia: Disorders of object recognition and what they tell us about normal vision. Cambridge: MIT Press.

Farah, M. J. (1991). Patterns of co-occurrence among the associative agnosias: Implications for visual object recognition. Cognitive Neuropsychology, 8, 1-19.

Farah, M. J. (1992). Is an object an object an object? Cognitive and neuropsychological investigations of domain specificity in visual object recognition. Current Directions in Psychological Science, 1, 164-169.

Farah, M. J. (1999). Relations among the agnosias. In G. W. Humphreys (Ed.), Case studies in the neuropsychology of vision (pp. 181-200). Hove, East Sussex: Psychology Press.

Farah, M. J. (2004). Visual agnosia (2nd ed.). Cambridge: MIT Press.

Farah, M. J., Rabinowitz, C., Quinn, G. E., \& Liu, G. T. (2000). Early commitment of neural substrates for face recognition. Cognitive Neuropsychology, 17, 117-123.

Gauthier, I., Behrmann, M., \& Tarr, M. J. (1999). Can face recognition really be dissociated from object recognition? Journal of Cognitive Neuroscience, 11, 349-370.

Gauthier, I., Behrmann, M., \& Tarr, M. J. (2004). Are Greebles like faces? Using the neuropsychological exception to test the rule. Neuropsychologia, 42, 1961-1970.

Gauthier, I., Curby, K. M., Skudlarski, P., \& Epstein, R. A. (submitted). Individual differences in FFA activity suggest independent processing at different spatial scales.

Gauthier, I., Curran, T., Curby, K. M., \& Collins, D. (2003). Perceptual interference supports a non-modular account of face processing. Nature Neuroscience, 6, 428-432.

Gauthier, I., Skudlarski, P., Gore, J. C., \& Anderson, A. W. (2000). Expertise for cars and birds recruits brain areas involved in face recognition. Nature Neuroscience, 3, 191-197.

Gauthier, I., \& Tarr, M. J. (1997). Becoming a "Greeble" expert: Exploring mechanisms for face recognition. Vision Research, 37, 1673-1682.

Gauthier, I., \& Tarr, M. J. (2002). Unraveling mechanisms for expert object recognition: Bridging brain activity and behavior. Journal of Experimental Psychology: Human Perception and Performance, 28, 431-446.

Gauthier, I., Tarr, M. J., Anderson, A. W., Skudlarski, P., \& Gore, J. C. (1999). Activation of the middle fusiform "face area" increases with expertise in recognizing novel objects. Nature Neuroscience, 2, 568-573.

Gauthier, I., Williams, P., Tarr, M. J., \& Tanaka, J. (1998). Training "greeble" experts: A framework for studying expert object recognition processes. Vision Research, 38, 2401-2428.

Genovese, C. R., Lazar, N. A., \& Nichols, T. (2002). Thresholding of statistical maps in functional neuroimaging using the false discovery rate. Neuroimage, 15, 870-878.

Grill-Spector, K., Knouf, N., \& Kanwisher, N. (2004). The fusiform face area subserves face perception, not generic within-category identification. Nature Neuroscience, 7 , 555-562.

Grill-Spector, K. (2003). The neural basis of object recognition Current Opinion in Neurobiology, 13, 159-166.

Hasegawa, I., \& Miyashita, Y. (2002). Categorizing the world: Expert neurons look into key features. Nature Neuroscience, 5, 90-91.

Haxby, J. V., Gobbini, M. I., Furey, M. L., Ishai, A., Schouten, J. L., \& Pietrini, P. (2001). Distributed and overlapping representations of faces and objects in ventral temporal cortex. Science, 293, 2425-2429. 
Humphreys, G. W., \& Rumiati, R. I. (1998). Stimulus specificity in visual recognition: Agnosia without prosopagnosia or alexia. Cognitive Neuropsychology, 15, 243-278.

Ishai, A., Ungerleider, L. G., Martin, A., Schouten, J. L., \& Haxby, J. V. (1999). Distributed representations of objects in the human ventral visual pathway. Proceedings of the National Academy of Sciences, U.S.A., 96, 9379-9384.

Kanwisher, N. (2000). Domain specificity in face perception. Nature Neuroscience, 3, 759-763.

Kanwisher, N., McDermott, J., \& Chun, M. M. (1997). The fusiform face area: A module in human extrastriate cortex specialized for face perception. Journal of Neuroscience, 17, 4302-4311.

Kolb, B., Gibb, R., \& Robinson, T. E. (2003). Brain plasticity and behavior. Current Directions in Psychological Science, 12, $1-5$.

Marotta, J. J., Behrmann, M., \& Genovese, C. (2001). A functional MRI study of face recognition in patients with prosopagnosia. NeuroReport, 12, 959-965.

Maurer, D., Le Grand, R., \& Mondloch, C. J. (2002). The many faces of configural processing. Trends in Cognitive Sciences, 6, 255-260.

McCarthy, G., Puce, A., Gore, J. C., \& Allison, T. (1997). Face-specific processing in the human fusiform gyrus. Journal of Cognitive Neuroscience, 5, 605-610.

McClelland, J. L., McNaughton, B. L., \& O'Reilly, R. C. (1995). Why are there complementary learning systems in hippocampus and cortex: Insights from learning and memory. Psychological Review, 102, 419-457.

Perrett, D. I., \& Oram, M. W. (1993). Neurophysiology of shape processing. Image and Vision Computing, 11, 317-333.

Pietrini, P., Furey, M. L., Ricciardi, E., Gobbini, M. I., Wu, W. H., Cohen, L., Guazzelli, M., \& Haxby, J. V. (2004). Beyond sensory images: Object-based representation in the human ventral pathway. Proceedings of the National Academy of Sciences, U.S.A., 101, 5658-5663.

Pizzamiglio, L., Galati, G., \& Committeri, G. (2001). The contribution of functional neuroimaging to recovery after brain damage: A review. Cortex, 37, 11-31.

Pleger, B., Foerster, A. F., Widdig, W., Henschel, M., Nicolas, V., Jansen, A., Frank, A., Knecht, S., Schwenkreis, P., \& Tegenthoff, M. (2003). Functional magnetic resonance imaging mirrors recovery of visual perception after repetitive tachistoscopic stimulation in patients with partial cortical blindness. Neuroscience Letters, 335, 192-196.

Rhodes, G., Byatt, G., Michie, P. T., \& Puce, A. (2004). Is the fusiform face area specialized for faces, individuation, or expert individuation? Journal of Cognitive Neuroscience, 16, 189-203.

Riddoch, M. J., \& Humphreys, G. W. (1993). Birmingham Object Recognition Battery. Hove, UK: Erlbaum.

Riddoch, M. J., \& Humphreys, G. W. (2003). Visual agnosia. Neurologic Clinics, 21, 501-520.

Riecker, A., Wildgruber, D., Grodd, W., \& Ackermann, H. (2002). Reorganization of speech production at the motor cortex and cerebellum following capsular infarction: A follow-up magnetic resonance imaging study. Neurocase, 8 417-423.

Rosen, H. J., Petersen, S. E., Linenweber, M. R., Snyder, A. Z., White, D. A., Chapman, L., Dromerick, A. W., Fiez, J. A., \& Corbetta, M. D. (2000). Neural correlates of recovery from aphasia after damage to left inferior frontal cortex. Neurology, 2000, 12.

Rossion, B., Caldara, R., Seghier, M., Schuller, A. M., Lazeyras, F., \& Mayer, E. (2003). A network of occipito-temporal face-sensitive areas besides the right middle fusiform gyrus is necessary for normal face processing. Brain, 126, 2381-2395.

Rumiati, R. I., \& Humphreys, G. W. (1997). Visual object agnosia without alexia or prosopagnosia: Arguments for separate knowledge stores. Visual Cognition, 4, 207-218.

Rumiati, R. I., Humphreys, G. W., Riddoch, M. J., \& Bateman, A. (1994). Visual object agnosia without prosopagnosia or alexia: Evidence for hierarchical theories of visual recognition. Visual Cognition, 1, 181-225.

Schwartz, S., Maquet, P., \& Frith, C. (2002). Neural correlates of perceptual learning: A functional MRI study of visual texture learning. Proceedings of the National Academy of Sciences, U.S.A., 99, 17137-17142.

Seniow, J., Polanowska, K., Mandat, T., \& Laudanski, K. (2003). The cognitive impairments due to the occipito-parietal brain injury after gunshot. A successful neurorehabilitation case study. Brain Injury, 17, 701-713.

Sergent, J., Ohta, S., \& MacDonald, B. (1992). Neuroanatomy of face and object processing. Brain, 115, 15-36.

Small, S. L., Flores, D. K., \& Knoll, D. C. (1998). Different neural circuits subserve reading before and after therapy for acquired dyslexia. Brain and Language, 62, 298-308.

Tarr, M. J., \& Cheng, Y. D. (2003). Learning to see faces and objects. Trends in Cognitive Neuroscience, 7, 23-30.

Tarr, M. J., \& Gauthier, I. (2000). FFA: a flexible fusiform face area for subordinate-level visual processing automatized by expertise. Nature Neuroscience, 3, 764-769.

Temple, E., Deutsch, G. K., Poldrack, R. A., Miller, S. L., Tallal, P., Merzenich, M. M., \& Gabrieli, J. D. E. (2003). Neural deficits in children with dyslexia ameliorated by behavioral remediation: Evidence from functional MRI. Proceedings of the National Academy of Sciences, U.S.A., 100, 2860-2865.

Thomas, R. M., Forde, E. M. E., Humphreys, G. W., \& Graham, K. S. (2002). A longitudinal study of category-specific agnosia. Neurocase, 8, 466-479.

Thulborn, K. R., Carpenter, P. A., \& Just, M. A. (1999). Plasticity of language-related brain function during recovery from stroke. Stroke, 30, 749-754.

Tsao, D. Y., Freiwald, W. A., Knutsen, T. A., Mandeville, J. B., \& Tootell, R. B. H. (2003). Faces and objects in macaque cerebral cortex. Nature Neuroscience, 6, 989-995.

Warburton, E., Price, C. J., Swinburn, K., \& Wise, R. J. S. (1999). Mechanisms of recovery from aphasia: Evidence from positron emission tomography studies. Journal of Neurology, Neurosurgery and Psychiatry, 66, 155-161. 


\section{AUTHOR QUERIES}

\section{AUTHOR PLEASE ANSWER QUERIES}

During the preparation of your manuscript, the questions listed below arose. Kindly supply the necessary information.

1. Results, Pretraining and Posttraining Imaging Comparison, S.M.: Please provide the $\chi^{2}$ value in $\chi^{2}$, $p<.05$.

2. Please update data in case there is a change in status:

- Caldara et al., submitted

- Gauthier et al., submitted

3. Cited in text but not in Reference list:

- Bukach et al., in preparation

\section{END OF ALL QUERIES}

